\title{
Radiation-induced Esophagitis in Lung Cancer-A Common Problem with Limited Therapeutic Options
}

\author{
Lawrence Berk \\ University of South Florida, Tampa, FL, USA
}

DOI: https://doi.org/10.17925/OHR.2021.16.2.95

$\mathrm{R}$ adiation-induced esophagitis is a common toxicity of radiation therapy of the chest, characterized by disruption of the epithelium due to damage to the underlying basal cells. The frequency of radiation-induced esophagitis correlates with the dose of radiation the esophagus receives and the volume that is treated with that dose. Modern radiation techniques are decreasing the size of the treatment field, potentially reducing the incidence and severity of esophagitis; however, it remains an important concern in patients with lung cancer treated with chemoradiotherapy. The pathogenesis of esophagitis is similar to that of head and neck mucositis, but there have been very few trials of treatments for the prevention of radiation-induced esophagitis. Current research is limited to several natural products which have shown activity in small randomized trials. However, several agents are currently being tested in clinical trials for the treatment of radiation-induced esophagitis. A successful agent to reduce/prevent esophagitis may allow higher doses of radiation to be delivered to the cancer and increase the curability of localized lung cancer. This review highlights the current understanding and limitations of data in radiation-induced esophagitis and current and potential future approaches to treatment.

\section{Keywords}

Radiation-induced esophagitis, esophagitis in lung cancer, treatment of esophagitis, radiation toxicities, non-small cell lung cancer

Disclosures: Lawrence Berk or his institution have received funding from Galera Therapeutics, Inc., as the principal investigator of phase lla clinical trial of avasopasem manganese (GC4419) for radiation-induced esophagitis in patients with non-small cell lung cancer.

Acknowledgments: Editorial support was provided by Sarah Hummasti, PHD, of AOIC, LLC, and was funded by Galera Therapeutics, Inc.

Review Process: Double-blind peer review.

Compliance with Ethics: This study involves a review of the literature and did not involve any studies with human or animal subjects performed by the author.

Authorship: The named author meets the International Committee of Medical Journal Editors (ICMJE) criteria for authorship of this manuscript, takes responsibility for the integrity of the work as a whole, and has given final approval for the version to be published.

Access: This article is freely accessible at touchONCOLOGY.com (c) Touch Medical Media 2020.

Received: July 20, 2020

Accepted: November 2, 2020

Published Online: December 16, 2020

Citation: Oncology \& Hematology Review. 2020;16(2):95-9

Corresponding Author: Lawrence Berk,

University of South Florida, Tampa General

Hospital Cancer Center, 3 Tampa General Circle,

Tampa, FL 33606, USA. E: berkl@usf.edu

Support: Editorial support was provided by AOIC LLC and funded by Galera Therapeutics, Inc.
Radiation-induced esophagitis, caused by incidental damage to the mucosal lining of the esophagus during radiation therapy, is a common and clinically important toxicity in patients with lung cancer Esophagitis generally develops $2-3$ weeks after initiation of radiation therapy and presents as pain and

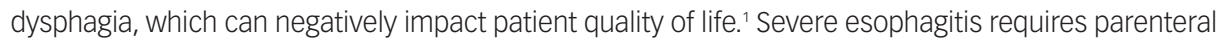
nutrition and can lead to hospitalization and treatment interruptions to allow for healing. ${ }^{2,3}$ Therefore, strategies to prevent radiation-induced esophagitis are of paramount importance to increase tolerance to radiation therapy and enable continuation of treatment to improve tumor outcomes.

Reports of the incidence of esophagitis are inconsistent due to lack of standardized methods to measure esophagitis as well as differences in the treatment regimen utilized (i.e., sequential or concurrent chemotherapy, radiation technique, radiation dose, and volume of tissue irradiated). ${ }^{1,4,5}$ In patients treated with concurrent chemotherapy and radiation therapy, which is the current standard of care for patients with non-small cell lung cancer (NSCLC), severe esophagitis occurs in up to onethird of patients. ${ }^{6.7}$ Advanced radiation techniques and models to identify predictors of esophagitis are being developed with the goal of reducing the incidence and severity of esophagitis. ${ }^{5,8-17}$ However, radiation-induced esophagitis remains an important limitation for radiation dose escalation efforts aimed at improving survival in patients with lung cancer. ${ }^{4}$ There is minimal utilization of radiation therapy alone for definitive treatment of lung cancer; therefore, this article will focus on the current standard of care for patients with lung cancer, rather than chemotherapy and radiation therapy separately.

This review provides a general overview of radiation-induced esophagitis in patients with lung cancer and is not intended to be a comprehensive review of the literature. Rather, the literature was surveyed to provide a concise summary of current knowledge of the pathogenesis of radiation-induced esophagitis in patients with lung cancer and how it is measured, as well as an overview of models to predict the incidence of radiation-induced esophagitis and the current treatment landscape. Incidence of esophagitis in other disease states, for example metastases, and other radiation-drug interactions, such as tyrosine kinase inhibitors, which are rarely used during thoracic irradiation, are not included in the scope of this review. 
Table 1: Grading scales for esophagitis

\begin{tabular}{|c|c|c|}
\hline & CTCAE Version $5.0^{23}$ & RTOG Scale ${ }^{24}$ \\
\hline Grade 1 & $\begin{array}{l}\text { Asymptomatic; clinical or diagnostic observations only; intervention } \\
\text { not indicated }\end{array}$ & $\begin{array}{l}\text { Mild dysphagia or odynophagia, requiring topical anesthetic, non-narcotic agents } \\
\text { or soft diet }\end{array}$ \\
\hline Grade 2 & Symptomatic; altered eating/swallowing; oral supplements indicated & Moderate dysphagia or odynophagia, requiring narcotic agents or liquid diet \\
\hline Grade 3 & $\begin{array}{l}\text { Severely altered eating/swallowing; tube feeding, TPN, or } \\
\text { hospitalization indicated }\end{array}$ & $\begin{array}{l}\text { Severe dysphagia or odynophagia with dehydration or weight loss (>15\% of } \\
\text { pretreatment baseline), requiring nasogastric feeding }\end{array}$ \\
\hline Grade 4 & Life-threatening consequences; urgent operative intervention indicated & Complete stricture, ulceration, perforation, or fistula \\
\hline Grade 5 & Death & Death \\
\hline
\end{tabular}

CTCAE = Common Terminology Criteria for Adverse Events; $R T O G=$ Radiation Therapy Oncology Group; TPN = total parenteral nutrition

\section{Pathophysiology of mucositis}

Radiation-induced esophagitis is much less studied than oral mucositis; however, the mucosa and the submucosa of the oral cavity and oropharynx are very similar to that of the esophagus up to the gastro-esophageal junction. ${ }^{18}$ Both are lined by keratinized and non-keratinized squamous epithelial cells overlying the lamina propria and the submucosa, which serve to protect underlying tissue. The esophagus has the same overall structure, but with muscularis mucosae between the lamina propria and the submucosa. As such, the pathophysiology of mucositis in both the oral cavity and esophagus is similar.

The current, generally accepted model outlining the five stages of mucositis-initiation, upregulation/activation, signal amplification, ulceration, and healing - was put forward by Sonis and colleagues and is relevant for both oral mucositis and esophagitis. ${ }^{19-22}$ In the initial phase, damage to epithelial cells occurs as a result of radiation therapy and/or chemotherapy during oncology treatment. ${ }^{20}$ Radiation induces doublestranded DNA breaks as a result of both direct damage to DNA and indirect damage caused by the generation of reactive oxygen species (ROS). In the second stage, release of ROS and other endogenous chemotherapy-related molecular patterns (CRAMPS) or damage-associated molecular patterns (DAMPS) from epithelial cells damaged in the first stage initiates a primary response that includes fibronectin breakdown, macrophage-mediated activation of matrix metalloproteinases, influx of inflammatory cells, and release of cytokines that further induce local tissue damage. Subsequently, a signal amplification stage occurs, which results in increased tissue damage and apoptosis through activation of nuclear factor (NF)-k $\beta$, tumor necrosis factor (TNF)- $\alpha$, and other cytokines, which results in a positivefeedback loop.

Among the cells damaged during these processes are the basal epithelial cells that generate the overlying mature squamous cells. The overlying squamous cells are more resistant to the effects of radiation therapy but continue to shed normally over time. The damage to the basal cells results in an insufficient supply of mature squamous cells, which in turn causes the surface of the epithelium to break down. The loss of the epithelial layer of mature squamous cells starts the next stage of the development of mucositis: ulceration and the loss of epithelia integrity. This gradual Ioss of the overlying cells without available replacement cells leads to a 2-week delay from the start of radiation therapy to when clinically evident mucositis sets in. The final stage of mucositis, healing, occurs spontaneously as a result of epithelial cell proliferation, differentiation, and migration from the wound margins in response to signaling from the extracellular matrix. During radiation therapy, the damaging insult is given daily, thus delaying the healing stage until after the radiation therapy is complete, with mucositis generally resolving 2-3 weeks after cessation of radiation therapy. There are currently no data available that directly correlate esophagitis with outcomes in patients with lung cancer, and so the detrimental effect of esophagitis on cure and control rates for these patients is unknown.

\section{Measurement of esophagitis}

No validated, standard measurement for esophagitis exists. Currently, esophagitis is generally scored using subjective measures, which are imprecise by nature. The most common are the National Cancer Institute's Common Terminology Criteria for Adverse Events (CTCAE) ${ }^{23}$ and the Radiation Therapy Oncology Group (RTOG) Scale (Table 1)..24 Both are observer rather than patient-scored scales and are arbitrary classifications for esophagitis. These grading scales do not represent true numerical values, and therefore scores cannot be added or averaged. Rather than $1-5$, they could equally be called $A-E$, more appropriately showing that non-parametric statistics must be used with these measures.

A modification of the CTCAE is the Patient-Reported Outcomes version of the Common Terminology Criteria for Adverse Events (PRO-CTCAE), designed to measure the patient experience of symptoms rather than proxy measurement by someone else. ${ }^{25}$ However, patients' reports of the severity of symptoms of esophagitis are not expected to be uniform between patients and may not even be reproducible in the individual patient because many outside factors can influence the perception of esophagitis, such as fatigue or depression. For clinical research, the MD Anderson Symptom Inventory for Lung Cancer scale, which assesses general symptoms during the treatment of lung cancer (13 of which are common to tumors at all sites and 3 that are specific to lung cancer), has also been used to measure toxicity during radiation therapy, but this measure is not specific to esophagitis. ${ }^{26,27}$ The core symptoms are pain, fatigue (tiredness), nausea, disturbed sleep, distress, shortness of breath, difficulty remembering, lack of appetite, drowsiness, dry mouth, feeling sad, vomiting, and numbness or tingling; and the 3 added symptoms are coughing, constipation, and sore throat, each patient reported on a 0-10 scale. Applying these measures directly to esophagitis requires assuming that reports of pain (the primary clinical symptom of esophagitis) and perhaps nausea are due to esophagitis.

Clinical assessment of radiation-induced esophagitis, including ruling out other causes of esophagitis, such as candida and viral infection, often requires more objective measures. Objective measurement of esophagitis through direct observation of the esophagus requires multiple endoscopies. 
This is not a practical or accurate method of measuring acute esophagitis, and is not recommended. To replace direct observation, the ability to objectively measure esophagitis utilizing imaging techniques has been examined. Studies showed that measuring esophageal expansion or cross-sectional areas on serial computerized tomography (CT) scans during radiation therapy can be used to quantify radiation-induced injury and correlated with the subjective measurement of esophagitis. ${ }^{28}$ Positron emission tomography (PET) scanning has also been examined for a potential correlation with subjective grades of esophagitis. In one study, the increase in standardized uptake values at 2 weeks correlated with grade 3 esophagitis. ${ }^{14}$ Another study found fluorodeoxyglucose (FDG) uptake was highly correlated with esophagitis grade.29

\section{Predictors of esophagitis during radiation therapy}

Esophagitis occurs during radiation therapy because part of the esophagus is incidentally irradiated during treatment. Tumor location adjacent to the esophagus or mediastinal lymph node involvement increases the risk of esophageal mucosa falling within the radiation field. The dose of radiation therapy that a structure, such as the esophagus, receives is reported as a dose-volume histogram. During treatment planning, the dose to the esophagus along its course is calculated as the percentage of the esophagus that will receive a specified dose and is reported as: $D_{\text {dose }}=$ volume (\%). For example, $D_{30}=50$ states that $50 \%$ of the esophagus receives 30 Gy of radiation.

A number of studies have examined predictors of radiation-induced esophagitis, and have linked female sex, low body mass index, pretreatment dysphagia, higher nodal stage, and concurrent chemotherapy to higher incidence of esophagitis. ${ }^{15,7,9,11,12,30,31}$ The impact of radiation technique and dosimetric factors on the incidence of esophagitis has also been examined. For example, a 2012 study by Gomez and colleagues on predictors of high-grade esophagitis after different techniques of radiation therapy (3D-conformal radiation therapy [CRT], intensity-modulated radiation therapy [IMRT], and proton beam therapy) for NSCLC found the incidence of severe esophagitis (grade $\geq 3$ using the CTCAE scale) correlated more closely with maximum esophagus dose than with the mean dose to the esophagus, highlighting the importance of weighing the percentage of esophagus receiving a high dose when evaluating treatment plans.? The study also found the incidence of grade $\geq 3$ esophagitis was highest with IMRT (28\% versus $8 \%$ [3D-CRT] and 6\% [proton beam therapy]), although other studies have shown similar incidence of esophagitis with IMRT and 3D-CRT. ${ }^{67,32,33} \mathrm{~A}$ study by Huang et al., modeling the relationship between esophagus dose-volume parameters and risk of grade $\geq 2$ esophagitis, found that the percent of the esophagus receiving at least 40 Gy was more closely correlated with the rate of toxicity than the mean dose..$^{34}$ In contrast, in patients treated with low-modulated IMRT, the mean esophageal dose correlated well with toxicity, with a mean esophageal dose of 15 Gy correlated with a $50 \%$ risk of grade 1 esophagitis (measured using the older RTOG scale) and a mean dose of 46 Gy correlated with a $50 \%$ risk of grade 2 esophagitis. ${ }^{35}$

Attempts to identify radiation technique and dosimetry-related factors predictive for esophagitis have failed to establish consensus on the optimal dose thresholds or relative importance of different predictors. $5,7,12,3,3,35$ Limited sensitivity of measurements to assess esophagitis and the use of different endpoints in different studies (i.e., CTCAE versus RTOG) complicate comparison between studies. In addition, most models to date examining predictors of esophagitis have looked at the incidence of grade $\geq 2$ esophagitis, due to the low risk of grade $\geq 3$ events. A recent study of 202 consecutive patients treated for NSCLC with radiation therapy utilized 35 clinical factors in a multivariate analysis performed by 11 machinelearning algorithms in an attempt to validate and rank predictors of grade $\geq 3$ esophagitis. ${ }^{12}$ The rate of grade $\geq 3$ esophagitis was $11 \%$ in the study; however, no reliable predictive models for severe esophagitis were identified. Additional studies are needed to establish reliable and consistent predictors for grade $\geq 3$ esophagitis to facilitate identification of patients most at risk for this toxicity, and to aid development of treatment strategies to mitigate radiation-induced esophagitis.

\section{Treatment of radiation-induced esophagitis}

Currently, no standard method for preventing or treating radiation-induced esophagitis exists. Although the Multinational Associate of Supportive Care in Cancer and the International Society of Oral Oncology have published joint, evidence-based recommendations for the management of mucositis secondary to cancer therapy, most of these apply to oral mucositis. ${ }^{36}$ For example, they cite strong evidence for the treatment of radiation-induced mucositis with benzydamine mouthwash, and weaker evidence supporting the use of treatments such as low-level laser therapy, morphine mouth rinses, doxepin mouth rinses, and zinc supplements to prevent radiationinduced mucositis. However, there are no studies supporting the use of these treatments for patients with esophagitis, and they would be technically difficult to implement to treat the esophagus.

Several compounds have been evaluated in clinical trials for the prevention or treatment of radiation-induced esophagitis, with conflicting results. In early studies, amifostine, a scavenger of free radicals produced during radiation therapy, showed mixed results as a potential radioprotective agent; ${ }^{37-41}$ however, no reduction in severe esophagitis was observed in a phase III study in patients with NSCLC on chemoradiotherapy (30\% incidence of grade $\geq 3$ esophagitis based on CTCAE criteria with amifostine versus $34 \%$ with control, $\mathrm{p}=0.9$ ). ${ }^{42}$ Sucralfate, a treatment for gastroduodenal ulcers, provided significant relief of esophagitis symptoms within 7 days (80\% versus $10 \%$ ) and accelerated tumor healing compared with treatment with the antacid sodium alginate in patients with esophageal cancer treated with radiation therapy. ${ }^{43}$ However, a separate trial found no substantial benefit of sucralfate versus placebo with regard to esophagitis and increased gastrointestinal toxicity (58\% of sucralfate-treated patients versus $14 \%$ of placebo patients; $p>0.0001$ ) and study drug discontinuation ( $40 \%$ for sucralfate and $4 \%$ for placebo) in the sucralfate group. ${ }^{44}$ Sodium alginate has also been studied as a potential treatment for esophagitis in patients treated with chemoradiotherapy for NSCLC. ${ }^{45}$ Rates of severe esophagitis (CTCAE grade $\geq 3$ ) were $12.8 \%$ in patients treated with sodium alginate at the start of chemoradiotherapy, $9.8 \%$ in patients treated with sodium alginate at onset of esophagitis, and $19.4 \%$ in the control group; however, differences were not statistically significant.

Several natural products have been evaluated in small, randomized, clinical trials for the prevention of radiation-induced esophagitis. Multiple studies have shown that honey can reduce radiation-induced oral mucositis suggesting a potential therapeutic role for patients with esophagitis as well. ${ }^{46}$ However, a randomized trial of Manuka honey (liquid or lozenges) versus supportive care found no difference in patient-reported pain with swallowing (numerical rating pain scale) at 4 weeks in patients receiving chemoradiotherapy for NSCLC. ${ }^{47}$ 
Whether an effect might be found using different treatment protocols or sources of honey remains to be determined. Studies with epigallocatechin-3gallate (EGCG), a compound extracted from green tea, have shown promise for the treatment of esophagitis. In an open-label, phase II study in patients with esophageal cancer receiving chemoradiotherapy or definitive radiation therapy, RTOG esophagitis scores decreased after EGCG treatment. ${ }^{48}$ Further, in a trial of 83 patients being treated for lung cancer, both prophylactic use of EGCG (at the start of chemoradiotherapy) and therapeutic application (at onset of esophagitis) resulted in a reduction in maximum acute esophagitis grade versus standard of care, with a slightly better effect with prophylactic application. ${ }^{49}$

Oral glutamine, which may play a role in tissue healing in response to damage from chemotherapy and radiation, has been shown to significantly reduce the severity of oral mucositis in patients treated with chemoradiotherapy for head and neck, suggesting the potential for a similar effect for esophagitis. ${ }^{50-52}$ In a single-center, randomized trial of 60 patients treated with chemotherapy and radiation therapy for NSCLC, glutamine treatment was associated with significantly less grade $2 / 3$ esophagitis (6.7\% versus $53.3 \%$ ) and delayed grade 2/3 onset (median 18.2 days versus 12.4 days) compared with the control arm..$^{53}$ The Chinese medicine Zhuye Shigao Granules has also been shown to significantly reduce the incidence of grade 2 esophagitis (9\% versus 23\%) in patients receiving concurrent or sequential chemoradiotherapy for lung, esophagus, or mediastinal cancer. ${ }^{54}$ Finally, whole course nutrition management, utilizing a complex nutritional plan based on a detailed nutritional assessment of the patient with diet monitored and adjusted as needed throughout treatment, significantly reduced maximum esophagitis grade $\geq 2$ (20\% in the nutritional management arm and $43 \%$ in the control arm). ${ }^{55}$
In addition to drug- and nutritional-based interventions to treat esophagitis, strategies to reduce incidental irradiation of the esophagus have been explored. However, in a randomized trial comparing target volume reduction informed by ${ }^{18} \mathrm{~F}-\mathrm{FDG}$ PET compared with conventional targeting, the rate of grade $\geq 3$ esophagitis or dysphagia was $16 \%$ in both arms..$^{15}$

Several active trials on ClinicalTrials.gov (as of April 15, 2020) for the prevention of esophagitis during chemotherapy and radiation therapy for lung cancer are ongoing. These include additional studies testing EGCG and oral nutritional supplements. Novel agents being tested include mecapegfilgrastim, a biosimilar to recombinant human granulocyte-colony stimulating factor (rhG-CSF), and avasopasem manganese (GC4419), a small molecule superoxide dismutase mimetic shown to reduce the duration, severity, and incidence of radiation-induced severe oral mucositis in patients with head and neck cancer undergoing radiation therapy. ${ }^{56}$

\section{Conclusion}

Despite advances in radiation therapy, esophagitis remains an important clinical concern in patients treated with concurrent chemotherapy and radiation therapy for lung malignancies. Dose escalation efforts to improve survival rates in patients with lung cancer have been limited by this and other radiation-induced toxicities. Efforts to improve and standardize objective measurement of esophagitis and development of predictive models to inform risk will be beneficial in developing strategies to mitigate the risk of severe esophagitis. Development of effective treatments for radiation-induced esophagitis could allow the use of higher doses of radiation when treating patients with lung cancer, potentially increasing cure rates. $\square$
1. Baker S, Fairchild A. Radiation-induced esophagitis in lung cancer. Lung Cancer (Auckl). 2016;7:119-27.

2. Cox JD, Pajak TF, Asbell S, et al. Interruptions of high-dose radiation therapy decrease long-term survival of favorable patients with unresectable non-small cell carcinoma of the lung: analysis of 1244 cases from 3 Radiation Therapy Oncology Group (RTOG) trials. Int J Radiat Oncol Biol Phys. 1993;27:493-8.

3. Deek MP, Kim S, Ahmed I, et al. Prognostic impact of missed chemotherapy doses during chemoradiation therapy for nonsmall cell lung cancer. Am J Clin Oncol. 2018;41:362-6.

4. Bradley JD, Paulus R, Komaki R, et al. Standard-dose versus highdose conformal radiotherapy with concurrent and consolidation carboplatin plus paclitaxel with or without cetuximab for patients with stage IIIA or IIIB non-small-cell lung cancer (RTOG 0617): a randomised, two-by-two factorial phase 3 study. Lancet Oncol. 2015:16:187-99.

5. Werner-Wasik M, Yorke E, Deasy J, et al. Radiation dosevolume effects in the esophagus. Int I Radiat Oncol Biol Phys. 2010:76:586-93.

6. Chun SG, Hu C, Choy H, et al. Impact of intensity-modulated radiation therapy technique for locally advanced non-small-cell lung cancer. a secondary analysis of the NRG Oncology RTOG 0617 randomized clinical trial. J Clin Oncol. 2017;35:56-62.

7. Gomez DR, Tucker SL, Martel MK, et al. Predictors of high-grade esophagitis after definitive three-dimensional conformal therapy, intensity-modulated radiation therapy, or proton beam therapy for non-small cell lung cancer. Int J Radiat Oncol Biol Phys. 2012;84:1010-6

8. Alam S, Thor M, Rimner A, et al. Quantification of accumulated dose and associated anatomical changes of esophagus using weekly magnetic resonance imaging acquired during radiotherapy of locally advanced lung cancer. Phys Imaging Radiat Oncol. 2020;13:36-43.

9. Hawkins PG, Boonstra PS, Hobson ST, et al. Prediction of radiation esophagitis in non-small cell lung cancer using clinical factors, dosimetric parameters, and pretreatment cytokine levels. Trans/ Oncol. 2018;11:102-8.

10. Huang EX, Bradley JD, El Naqa I, et al. Modeling the risk of radiation-induced acute esophagitis for combined Washington University and RTOG trial 93-11 lung cancer patients. Int J Radiat Oncol Biol Phys. 2012;82:1674-9.

11. Huang J, He T, Yang R, et al. Clinical, dosimetric, and position factors for radiation-induced acute esophagitis in intensitymodulated (chemo)radiotherapy for locally advanced non-smallcell lung cancer. Onco Targets Ther. 2018;11:6167-75.

12. Luna JM, Chao HH, Shinohara RT, et al. Machine learning highlights the deficiency of conventional dosimetric constraints for prevention of high-grade radiation esophagitis in non-small cell lung cancer treated with chemoradiation. Clin Transl Radiat Oncol. 2020;22:69-75

13. Ma L, Qiu B, Li Q, et al. An esophagus-sparing technique to limit radiation esophagitis in locally advanced non-small cell lung cancer treated by simultaneous integrated boost intensitymodulated radiotherapy and concurrent chemotherapy. Radiat Oncol. 2018;13:130

14. Mehmood Q, Sun A, Becker N, et al. Predicting radiation esophagitis using 18F-FDG PET during chemoradiotherapy for locally advanced non-small cell lung cancer. J Thorac Oncol. 2016;11:213-21.

15. Nestle U, Schimek-Jasch T, Kremp S, et al. Imaging-based target volume reduction in chemoradiotherapy for locally advanced non-small-cell lung cancer (PET-Plan): a multicentre, open-label, randomised, controlled trial. Lancet Oncol. 2020;21:581-92.

16. Niedzielski J, Bluett JB, Williamson RT, et al. Analysis of esophageal-sparing treatment plans for patients with high-grade esophagitis. J App/ Clin Med Phys. 2013;14:4248.

17. Yu Y, Guan $H$, Dong $Y$, et al. Advances in dosimetry and biological predictors of radiation-induced esophagitis. Onco Targets Ther. 2016;9:597-603.

18. Squier CA, Kremer MJ. Biology of oral mucosa and esophagus. J Natl Cancer Inst Monogr. 2001;(29):7-15.

19. Basile D, Di Nardo P, Corvaja C, et al. Mucosal Injury during anticancer treatment: from pathobiology to bedside. Cancers (Basel). 2019;11:857.

20. Maria OM, Eliopoulos N, Muanza T. Radiation-induced ora mucositis. Front Oncol. 2017;7:89.

21. Sonis ST. Pathobiology of mucositis. Semin Oncol Nurs. 2004;20:11-5.

22. Villa A, Sonis ST. Mucositis: pathobiology and management. Curr Opin Oncol. 2015:27:159-64

23. US Department of Health and Human Services, Common terminology criteria for adverse events (CTCAE) Version 5.0, 2017. Available at: https://ctep.cancer.gov/protocoldevelopment/ electronic_applications/docs/CTCAE_v5_Quick_Reference_5x7. pdf (accessed November 9, 2020)

24. Radiation Therapy Oncology Group. RTOG/EORTC late radiation morbidity scoring schema. Philadelphia, PA, USA: RTOG, 2020

25. Basch E, Pugh SL, Dueck AC, et al. Feasibility of patient reporting of symptomatic adverse events via the patient-reported outcomes version of the common terminology criteria for adverse events (PRO-CTCAE) in a chemoradiotherapy cooperative group multicenter clinical trial. Int I Radiat Oncol Biol Phys.
2017:98:409-18

26. Mendoza TR, Wang XS, Lu C, et al. Measuring the symptom burden of lung cancer: the validity and utility of the lung cance module of the M. D. Anderson Symptom Inventory. Oncologist. 2011;16:217-27

27. Wang XS, Shi Q, Williams LA, et al. Prospective study of patientreported symptom burden in patients with non-small-cell lung cancer undergoing proton or photon chemoradiation therapy. $\int$ Pain Symptom Manage 2016:51:832-8.

28. Niedzielski JS, Yang J, Stingo F, et al. Objectively quantifying radiation esophagitis with novel computed tomography-based metrics. Int J Radiat Oncol Biol Phys. 2016;94:385-93.

29. Niedzielski JS, Yang J, Liao Z, et al. (18)F-Fluorodeoxyglucose positron emission tomography can quantify and predict esophageal injury during radiation therapy. Int J Radiat Oncol Biol Phys. 2016;96:670-8.

30. Challand T, Thureau S, Dubray B, Giraud P. Esophageal toxicity of radiation therapy: clinical risk factors and management. [Article in French]. Cancer Radiother. 2012;16:364-71.

31. Patel AB, Edelman MJ, Kwok Y, et al. Predictors of acute esophagitis in patients with non-small-cell lung carcinoma treated with concurrent chemotherapy and hyperfractionated radiotherapy followed by surgery. Int I Radiat Oncol Biol Phys. 2004;60:1106-12.

32. Ling $\mathrm{DC}$, Hess $\mathrm{CB}$, Chen $\mathrm{AM}$, et al. Comparison of toxicity between intensity-modulated radiotherapy and 3-dimensional conformal radiotherapy for locally advanced non-small-cell lung cancer. Clin Lung Cancer. 2016;17:18-23.

33. Shirvani SM, Jiang J, Gomez DR, et al. Intensity modulated radiotherapy for stage III non-small cell lung cancer in the United States: predictors of use and association with toxicities. Lung Cancer. 2013;82:252-9.

34. Huang EX, Robinson CG, Molotievschi A, et al. Independent test of a model to predict severe acute esophagitis. Adv Radiat Oncol. 2017;2:37-43

35. He R, Hamadane E, Benghuzzi A. Dosimetric predictive model for esophagitis induced from radiotherapy of lung cancer patients. J Miss Acad Sci. 2018;63:233-6.

36. Lalla RV, Bowen J, Barasch A, et al. MASCC/ISOO clinical practice guidelines for the management of mucositis secondary to cancer therapy. Cancer. 2014;120:1453-61.

37. Antonadou D, Throuvalas N, Petridis A, et al. Randomized phase III trial of radiation treatment + /- amifostine in patients with advanced-stage lung cancer. Int J Radiat Oncol Biol Phys. 2001;51:915-22

38. Koukourakis MI, Kyrias G, Kakolyris S, et al. Subcutaneous 
administration of amifostine during fractionated radiotherapy: A randomized phase II study. J Clin Oncol. 2000;18:2226-33.

39. Komaki R, Lee JS, Milas L, et al. Effects of amifostine on acute inoperable non-small-cell lung cancer Report of a randomized comparative trial. Int J Radiat Oncol Biol Phys. 2004;58:1369-77.

40. Leong SS, Tan EH, Fong KW, et al. Randomized double-blind trial of combined modality treatment with or without amifostine in unresectable stage III non-small-cell lung cancer. J Clin Oncol. 2003;21:1767-74

41. Senzer N. A phase III randomized evaluation of amifostine in stage IIIA/IIIB non-small cell lung cancer patients receiving concurrent carboplatin, paclitaxel, and radiation therapy followed by gemcitabine and cisplatin intensification: Preliminary findings. Semin Oncol. 2002:29:38-41.

42. Movsas B, Scott C, Langer $\mathrm{C}$, et al. Randomized trial of amifostine in locally advanced non-small-cell lung cancer patients receiving chemotherapy and hyperfractionated radiation: radiation therapy oncology group trial 98-01. J Clin Oncol. 2005;23:2145-54.

43. Sur RK, Kochhar R, Singh DP. Oral sucralfate in acute radiation oesophagitis. Acta Oncol. 1994;33:61-3.

44. McGinnis WL, Loprinzi CL, Buskirk SJ, et al. Placebo-controlled trial of sucralfate for inhibiting radiation-induced esophagitis. I Clin Oncol. 1997:15:1239-43.
45. Yokoyama T, Ninomiya K, Oze I, et al. 1807P - A randomized tria of sodium alginate prevention of radiation-induced esophagitis in patients with locally advanced NSCLC receiving concurrent chemoradiotherapy: OLCSG1401. Ann Oncol. 2019:30:v734-5.

46. Liu TM, Luo YW, Tam KW, et al. Prophylactic and therapeutic effects of honey on radiochemotherapy-induced mucositis: a meta-analysis of randomized controlled trials. Support Care Cancer. 2019;27:2361-70.

47. Fogh SE, Deshmukh S, Berk LB, et al. A randomized phase 2 trial prophylactic manuka honey for the reduction of chemoradiation therapy-induced esophagitis during the treatment of lung cancer. results of NRG Oncology RTOG 1012. Int J Radiat Oncol Biol Phys. 2017;97:786-96.

48. Li X, Xing L, Zhang Y, et al. Phase II trial of epigallocatechin-3gallate in acute radiation-induced esophagitis for esophagus cancer. J Med Food. 2020;23:43-9.

49. Zhao $\mathrm{H}$, Jia $\mathrm{L}$, Chen $\mathrm{G}$, et al. A prospective, three-arm, randomized trial of EGCG for preventing radiation-induced esophagitis in lung cancer patients receiving radiotherapy. Radiother Oncol. 2019;137:186-91.

50. Anderson PM, Lalla RV. Glutamine for amelioration of radiation and chemotherapy associated mucositis during cancer therapy. Nutrients. 2020:12:E1675.

51. Chattopadhyay S, Saha A, Azam M, et al. Role of oral glutamine in alleviation and prevention of radiation-induced oral mucositis: a prospective randomized study. South Asian I Cancer. 2014;3:8-12.

52. Tsujimoto T, Yamamoto Y, Wasa M, et al. L-glutamine decreases the severity of mucositis induced by chemoradiotherapy in patients with locally advanced head and neck cancer: a double-blind, randomized, placebo-controlled trial. Oncol Rep. 2015;33:33-9.

53. Chang SC, Lai YC, Hung JC, et al. Oral glutamine supplements reduce concurrent chemoradiotherapy-induced esophagitis in patients with advanced non-small cell lung cancer. Medicine (Baltimore). 2019;98:e14463

54. Wang LJ, Lu JZ, Cai BN, et al. Effect of Compound Zhuye Shigao Granule () on acute radiation-induced esophagitis in cancer patients: a randomized controlled trial. Chin I Integr Med. 2017:23:98-104.

55. Qiu Y, You J, Wang K, et al. Effect of whole-course nutrition management on patients with esophageal cancer undergoing concurrent chemoradiotherapy: a randomized control trial. Nutrition. 2020;69:110558

56. Anderson CM, Lee CM, Saunders DP, et al. Phase IIb, randomized, double-blind trial of GC4419 versus placebo to reduce severe oral mucositis due to concurrent radiotherapy and cisplatin for head and neck cancer. I Clin Oncol. 2019:37:3256-65. 